\title{
Clinical audit in farm animal veterinary practice
}

\section{Part 1: Preparing for audit}

This article, the first in a two-part series, discusses the preparation and initiation of clinical audit in farm animal practice. In the second article, to be published in the xxx issue of In Practice, the stages of data collection, analysis and re-audit will be discussed along with some of the facilitators and barriers to conducting clinical audit. Both articles are based on findings from a three year research project looking at the use of clinical audit in farm animal practice. The research included conducting audits using information already in existence in the practice setting, and audits focused on actively collecting information over a period of time in three farm animal practices, and a nationwide survey on the experiences and attitudes of farm animal veterinary surgeons towards clinical audit in the UK.

\section{Clinical audit in farm animal practice}

Clinical audit in the veterinary setting is the assessment and improvement of clinical services delivered by veterinary practices and practitioners. Essentially, it's looking in detail at what you do and trying to make it better. Clinical audit is a continuous cycle that uses the best available evidence to improve the care delivered to patients (Figure 1). 


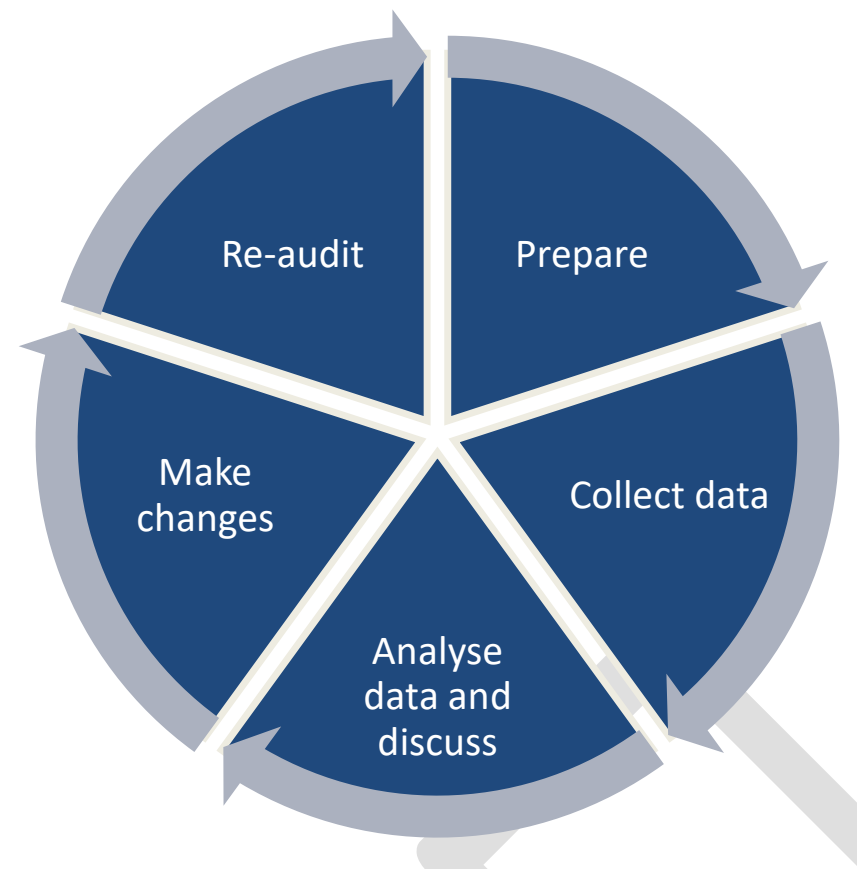

Figure 1 The clinical audit cycle (taken from Waine and Brennan 2015)

\section{Conducting clinical audit in farm practice for the first time:}

\section{- $\quad$ Keep it simple}

Where very little information currently exists or is collected, the undertaking of a clinical audit that provides any data will be of interest. Start simple - how do different vets do different things across the practice? How many of a certain case do you see? How much of a certain product do you sell? Difficult topics can discourage future clinical audit activity.

- Keep it short

Start with a short audit, regardless of whether you are using data already in existence within your practice, or whether you need to collect information going forwards. For example, your data collection forms should contain no more than 10 questions, and data should be collected for a short time (less than 3 months) if feasible. Avoid long audits that can lead to audit fatigue and communicate the results clearly to allow individuals to see the benefits.

- Keep it interesting

Choose a topic that the majority of the team have an interest in. Show the benefits that can be gained by collecting and analysing data simply. Aim for success rather than answers to too many complicated questions. 


\section{Preparing and initiating the clinical audit}

Preparation is the key to success and time spent on this stage should increase the chance of conducting a useful and effective clinical audit.

\subsection{Assign a lead}

An individual within the team needs to take ownership of the clinical audit to drive it forward within the practice. The lead can be any member of practice staff; veterinary assistant, office administrator, partner/director or veterinary nurse, and they may be junior or senior staff members. The lead position may be best suited to the person most enthused about the topic, therefore benefits may be felt from having a different audit lead for different clinical audit topics. It is important to ensure that whoever is leading the process is allocated suitable time to dedicate to facilitating the clinical audit. The clinical audit lead should oversee the entire process and ensure that it progresses, is completed and that results are fed back to the individuals involved in the practice. They should be in a position to motivate the team to carry out the clinical audit and ensure that new and returning staff members are aware of the clinical audit progress.

\subsection{Engage all team members in the process}

It is important to establish who will make up the clinical audit team (Viner 2005) and who will be involved. This may include veterinary surgeons, veterinary nurses, office admin staff and visiting veterinary students. Because farm animal practitioners are often working in isolation and may have limited contact with others in the practice on a day to day basis, it is even more important that engagement occurs during the early stages of the audit. 


\subsection{Ensure a no-blame culture from the start}

A no-blame culture is vital for successful clinical audits (Viner 2005; Viner and Jenner 2005; Oxtoby et al 2015). If practitioners are unable to feel comfortable discussing and sharing experiences, then clinical audit will have little benefit and fundamentally quality improvement should start at the level of improving communication within the practice team. The lead should be selected carefully when attempts are being made to run clinical audits for the first time so that the process is likely to be more successful. In practices where it is not possible for the whole team to do clinical audit, individuals may consider conducting clinical audits of their own activities.

\subsection{Determine resources available}

Before conducting a clinical audit, the lead should find out what resources are available within the practice and investigate the capabilities of the individuals who will be involved. This may be time available to dedicate to the process, knowledge and experience of team members, capabilities of the practice management system (PMS) software or the availability of other data or information held by the practice.

\subsection{Create clear communication strategies}

Successful audit requires successful communication (Rayment 2002). Clinical audit can have huge benefits in relation to the encouragement and enhancement of communication within a practice (Yagi 2015). Establish the channels that are available within the practice and determine which ones will allow all team members to easily stay up to date (e.g. via emails, memos, posters, PMS messages, notes in pigeon holes, WhatsApp groups etc.) at the beginning of the process. It is important not to allow communication to become over- 
bearing or time consuming. The frequency of contact will depend on the length of audit, method of communication and caseload seen.

\subsection{Choose a topic to audit}

Choose a topic to audit based on the interests of the team. The topic you choose may be something that has a high caseload, carries a high risk or involves a high cost, or it may be the topic from a recently attended continuing professional development (CPD) course, the subject of a newly published paper or an area you want to develop within the practice. Because farm animal clinicians are often working alone, they are less likely to observe other vets and potentially the variability in case approach, so without regular practice communication it is possible that individuals may be using very different techniques unknowingly. Therefore clinical audit discussions are important to highlight any areas of variability and the reasons behind these.

\subsubsection{Meeting to decide the clinical audit topic}

An initial meeting to discuss and choose the topic to focus on is likely to be beneficial and will kick start the process for the clinical audit lead. Staff involvement is crucial so the meeting should be held at a time that suits as many of the team as possible, or provide a way of individuals joining remotely (e.g. teleconference, Skype, Whatsapp). Incentives should be provided (such as food and drink) where staff are giving up their free time, or hold discussions during paid working hours if possible. The lead should ask everybody involved to suggest topics to audit prior to the meeting; this could be done via email, Facebook (private group), Whatsapp or a suggestions box.

During the meeting, the group should brain storm (Rayment 2002) from the original topics suggested to create more detailed choices if necessary. Figure 2 uses the broad topic of 'calf 
pneumonia' as an example to describe the different types of specific clinical audit that could be conducted. Not all topics will be amenable to every type of audit and it is likely that some topic ideas will be rejected at this stage - try to cut down to less than five for more detailed discussion. With the remaining five (or less) topics, the discussion should focus on the advantages and disadvantages of each topic. Things to consider could include: case load seen; the interest surrounding the case; how easy it will be to record, collect or find data and how useful the results may be. It may be pertinent to consider how seasonality may affect data collection (e.g. calf pneumonia, summer mastitis in cows, pregnancy toxaemia in ewes); running the clinical audit during the busiest period may allow maximum cases to be collected over the shortest period of time. The final topic to audit should then be chosen after all individuals have had an opportunity to contribute.

The discussion should allow all stakeholders to have a say and voice their opinions. Any concerns raised about the clinical audit need to be acknowledged and addressed before the process begins to avoid subsequent issues. The audit lead should ensure that the meeting stays on track and that the clinical audit discussion does not stray into a clinical research discussion (what the best way to do something is; see Figure 3). After the meeting, communicate the findings to everybody that will be involved in the clinical audit, including those members of staff unable to attend, or those that get called away during the meeting. 


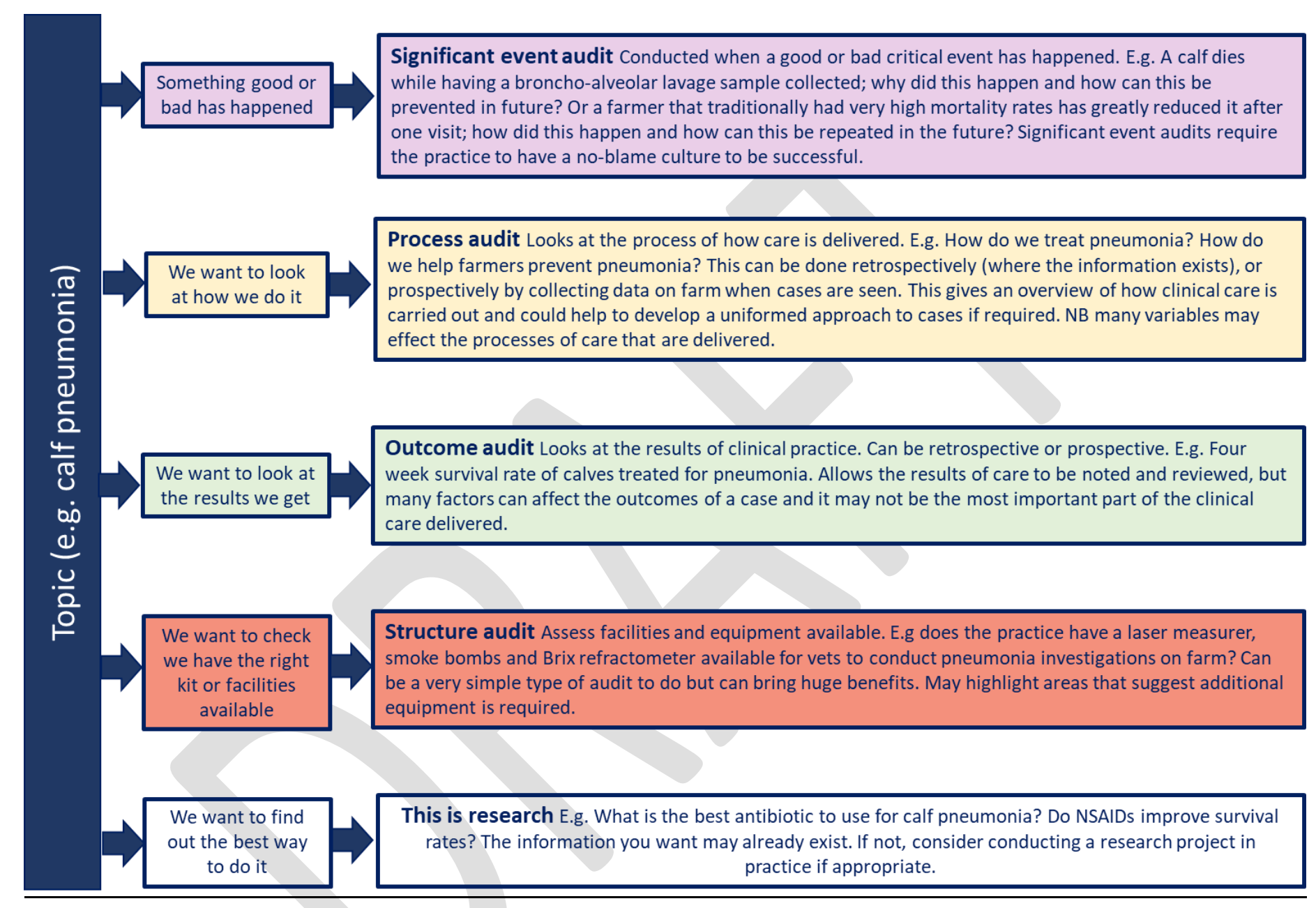

Figure 2 Possible examples of the different types of clinical audit that could be conducted arising from the decision to focus on the area of calf pneumonia 


\subsubsection{Search the literature}

Once the topic to audit has been chosen, the literature should be searched for any relevant evidence that may help you set a standard to work with or that provides more information on any recent developments in the area about how things should be done. Literature searches should be thorough with the aim of finding any existing standards, consensus guidelines or discussion of current techniques used in relation to the topic. Any information found may be useful to your clinical audit (Rayment 2002). For rapid determination of whether evidence exists on a topic, an initial search for existing structured reviews of the literature should be carried out first (see 'Evidence summaries' box).

\section{Evidence summaries}

There are a number of existing resources highlighting reviews of the literature that have already been conducted:

- BestBETs for Vets www.bestbetsforvets.org

- RCVS Knowledge: Knowledge summaries (https://www.veterinaryevidence.org/index.php/ve)

- VetSRev - Database of veterinary systematic reviews(http://webapps.nottingham.ac.uk/refbase/)

- Equine Veterinary Education: Clinical evidence in equine practice (https://onlinelibrary.wiley.com/page/journal/20423306/homepage/clinical evi dence in equine practice online collection.htm)

For primary searching, Grindlay et al. (2012) recommends searching Medline (PubMed) and $C A B$ Abstracts for the best coverage of veterinary journals. Finding references through relevant article citations or via Google Scholar may also yield results. The online EBVM Learning website has further details on how to search the literature (http://www.ebvmlearning.org). 


\section{Accessing the literature}

There are a number of different ways that the literature can be accessed:

- Some articles are Open Access and therefore can be freely obtained

- Individuals studying towards certificates or diplomas may have access to journals through the university they are enrolled with

- The RCVS Knowledge Library can be joined for an annual membership fee ( $£ 125 /$ year for veterinary surgeons, $£ 70 /$ year for veterinary surgeons less than 2 years qualified or $£ 50 /$ year for veterinary nurses at the time of writing)

- VetMed Resource (www.cabi.org/vetmedresource)

- Local libraries can have access to scientific journals

- In the UK, British Veterinary Association members have access to the Veterinary Record and In Practice; British Cattle Veterinary Association members can access Cattle Practice; British Equine Veterinary Association members have access to the Equine Veterinary Journal and Equine Veterinary Education journals; British Small Animal Veterinary Association members have access to VetMed Resource (see above) as part of their membership

\section{i. $\quad$ Setting a standard}

Traditionally, clinical audit compares practice against a set, evidence-based standard.

However, this is not always possible in veterinary medicine where evidence is often lacking.

There are a few approaches you can take, depending on the information that you find on your topic. For more detail in relation to this, see Table 2 in Waine and Brennan (2015).

a) If you have identified existing consensus guidelines you may want to use them as your standard to compare your current practice to, or use them to make your own local practice guidelines. For example, Wapenaar et al. (2011) published evidencebased guidelines on the management of uterine prolapse in cattle. You could:

i. Compare what you currently do in your practice to the guideline

ii. Implement the guideline in your practice and then compare how well they were followed by the team 
iii. Use the consensus guideline to create your own local practice guideline if you would like all vets to take a similar approach to managing uterine prolapses

b) You may have found previous literature that you could be useful to compare your practice to. For example, if doing an outcome audit looking at the survival of cows after surgery for a left displaced abomasum (LDA), the findings published by Pedersen (2006) could be used as a comparison. The use of studies in this way must be approached with caution, as certain aspects regarding how studies have been designed may mean direct comparison is difficult. If possible, a quality assessment of any literature found should be carried out. There are bespoke tools available to help vets to be able to make a judgement on study quality; please see Dean (2013) for further details.

c) You may not have found any relevant peer-reviewed evidence related to your clinical audit topic. If this is the case you could discuss the topic with the practice team and set your own benchmark to aim for. For example, perhaps the team decides that they would like $80 \%$ of caesareans to receive a post-op check within 48 hours. Alternatively, you could conduct the first round of the clinical audit to determine current activities and performance, and use the findings as a future basis to audit against.

\section{Research vs audit: Ethical considerations}

Ensure the clinical audit you want to do is not actually research. There can be a fine line between clinical audit and clinical research; research determines the right thing to do, while 
audit looks at what is being done (Smith 1992; Wylie 2015). Clinical audit should be no more than normal clinical management (Viner 2009) and not the identification of which approaches or treatments are the most efficacious. There are some features that are very clearly only applicable to clinical research: interventions using completely new treatments, an absolute requirement for ethical approval and a clearly defined methodology with repeatable results. However, this does not mean that ethical considerations should not be identified for clinical audits. Ensure methods maintain client confidentiality and meet the requirements of the Data Protection Act (1998) or after May 2018, the new General Data Protection Regulation (GDPR; Read 2018).

Some types of research studies, such as randomised controlled trials, are very clearly not clinical audit, but other types of research, such as retrospective case-control studies or a case series, may be harder to distinguish.

Dunn (2012) argues that the purpose of clinical audit is not to create new knowledge. However, when clinical audit is conducted and reported in an area where there is no existing evidence, new knowledge may be created. This may be where some of the confusion lies. Figure 3 shows a simplified example of how a research study and a clinical audit could ultimately produce the same data. Although the same data may be collected, the key difference between the two is how the process is approached and how the results are interpreted and used. New knowledge may be created from clinical audit, but it must be interpreted with care as clinical audit data are unlikely to be as robust as those generated from a well conducted research project.

The distinction and intention of a project should be clear from the outset as the process between research and clinical audit does differ. If work is appropriately labelled and 
understood the findings can be correctly interpreted for quality and subsequently utilised. This means clinical audit findings could be used as a reference for other work or used as a benchmark by others. However, this must be considered before you conduct your clinical audit. Ethical consideration must be undertaken if you plan to share data outside of your practice or you plan to publish your clinical audit on your website, in a newsletter or in a publication. Where clinical audits are published, publishing formats should ensure that the clinical audits are clearly described as such, and are not moulded into a research template which may lead to confusion. 
Research

Plan: A retrospective case-control study looking at a population of animals to determine which treatment is best for a given condition

\section{Clinical audit}

A retrospective outcome clinical audit to look at recovery times after patients have been treated by a practice for a given condition

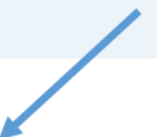

\section{Methods:}

Review the clinical records for animals treated for the given condition over a set time period

- Detailed, repeatable methodology required

- Thorough literature search to see what previous work exists

- Sample size calculation to determine number of cases needed

- Ethical approval required

- Detailed statistical analysis of results
Results:

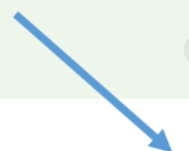

- Literature search to see if any research has been done that could provide a standard or target to compare results to No specific number of cases required

- Detailed, repeatable methodology not required

- Only basic, descriptive statistics needed

- Ethical approval not needed if not planning to share or publish results

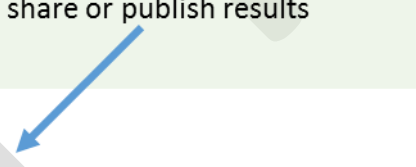

\section{Conclusion:}

Treatment B leads to quicker recovery times for the given condition than Treatment $A$.

- The study discussion may include how this is applicable to veterinary practice

- Study limitations should be discussed when findings reported

- Future research work may be suggested

\section{Use of}

findings: Treatment $B$ is better than Treatment $A$. Assuming a robust study, the findings could be used as a reliable reference.
$X$ clinical records found.

Y\% treated with Treatment A

Z\% treated with Treatment B

Treatment $A$ led to recovery in an average of 7 days

eatment $B$ led to recover in an average of 4 days
1

this practice animals treated with

Ireatment B appeared to recover more

quickly than those treated with Treatment A.

- Agreement with any existing literature should be assessed

I - Findings should be discussed within the practice and decisions made as to if any changes are necessary

Findings are relevant to the practice that conducted the audit. Results could be used as a benchmark for others BUT must remember that the results are from clinical audit and not research.

Figure 3 An example outlining the difference between research and clinical audit 


\section{Conclusion}

For the best chance of conducting a useful and successful clinical audit, adequate time should be spent on preparation. This will help the rest of the process to progress smoothly and increase the chances of the clinical audit being beneficial to all involved. As mentioned above, the second part of this series will contain the second part of clinical audit, including data collection, analysis and re-audit.

\section{Acknowledgements}

We would like to thank the 3 farm animal practices (including Bishopton Veterinary Group) for working with us as part of the research project that this article is based on. We would also like to thank all veterinary surgeons who participated in the clinical audit nationwide survey.

\section{References}

DEAN, R.S. (2013) How to read a paper and appraise the evidence. In Practice 35, 282-285

DUNN, J. (2012) Clinical audit: a tool in the defence of clinical standards. In Practice 34, 167169

GRINDLAY, D. J., BRENNAN, M. L. \& DEAN, R. S. (2012) Searching the veterinary literature: a comparison of the coverage of veterinary journals by nine bibliographic databases. J Vet Med Educ 39, 404-412

OXTOBY, C., FERGUSON, E., WHITE, K. \& MOSSOP, L. (2015) We need to talk about error: causes and types of error in veterinary practice. Veterinary Record 177, 438-444

PEDERSEN, S. (2006) Analysis of reproductive performance, milk production and survival following surgery for a left displaced abomasum in dairy cattle. Cattle Practice 14, 221-226

RAYMENT, K. (2002) Clinical audit - a means of evaluating 'quality'. In Practice 24, 481-484

READ, C. (2018) What the new data protection regulation means for veterinary practices. In Practice, 40, 75-77. 
SMITH, R. (1992) Audit and research. BMJ 305, 905-906

VINER, B. (2005) Clinical audit in veterinary practice - the story so far. In Practice 27, 215218

VINER, B. (2009) Using audit to improve clinical effectiveness. In Practice 31, 240-243

VINER, B. P. \& JENNER, C. S. (2005) Clinical audit - learning from the medical profession. Veterinary Record 157, 695-696

WAINE, K. \& BRENNAN, M.L. (2015) Clinical audit in veterinary practice: theory v reality. In Practice 37, 545-549

WAPENAAR, W., GRIFFITHS, H., LOWES, J. \& BRENNAN, M. (2011) Developing evidencebased guidelines using expert opinion for the management of uterine prolapse in cattle.

Cattle Practice 19, 17-21

WYLIE, C. E. (2015) Prospective, retrospective or clinical audit: A label that sticks. Equine Vet J 47, 257-259

YAGI, K. (2015) Has RECOVER changed us? A hospital's experience in implementing evidence-based guidelines. Available at: https://rcvsknowledge.conferenceservices.net/reports/template/onetextabstract.xml?xsl=template/onetextabstract.xsl\&conf erencelD=4065\&abstract ID=854171. Accessed 25 th March 2018. 